\title{
Retrospective study of factors related to preterm labor in Yasuj, Iran
}

This article was published in the following Dove Press journal:

International Journal of General Medicine

6 December 2012

Number of times this article has been viewed

\section{Seyed Hesamedin \\ Nabavizadeh' \\ Mohammad Malekzadeh ${ }^{2, *}$ \\ Ali Mousavizadeh' \\ Hamid Reza Ghaffarian \\ Shirazi' \\ Parvin Ghaffari' \\ Nooshin Karshenas ${ }^{3}$ \\ Tahmineh Malekzadeh ${ }^{3}$ \\ Mohammad Zoladl' \\ 'Social Determinants of Health Research Center, Yasuj University of Medical Sciences, Yasuj, Iran; \\ ${ }^{2}$ Department of Psychology, Osmania University, Hyderabad, India; \\ 'Students' Research Committee, Yasuj University of Medical Sciences, Yasuj, Iran}

Correspondence: Mohammad Malekzadeh Department of Psychology, Osmania University, Shivam Road, Prashanti Nagar, Nallakunta, Hyderabad, Andhra

Pradesh, India

$\mathrm{Tel}+9|72074| 5160$

Emailmzh54I@yahoo.com
Introduction: Premature labor is a serious worldwide problem that can cause neonatal death and other serious disorders. This study aimed to determine the most important factors related to preterm labor in Yasuj, Iran.

Method: This case-control study was conducted in the maternity ward of Imam Sajjad Hospital, the obstetrics and gynecology center of Yasuj, in 2010. Among eligible samples, mothers with preterm labor were selected as the case group, and for each sample in the case group, one mother with full-term labor was selected by using clipper-matched sampling to make up the control group. Data were collected by a researcher-made questionnaire and the 28 -item General Health Questionnaire. Finally, after deleting imperfect questionnaires, collected data of 52 subjects of case group and the same amount in control group were analyzed.

Results: Among the 5400 live birth infants in Yasuj in 2010, 130 infants were premature $(2.4 \%)$. The preterm labor risk in women with two or more pregnancies was 5.5 times more than women with less than two pregnancies, its risk in women with low general health status was 2.9 times more than in women with normal general health status, and the preterm labor risk in women with a history of diabetes mellitus/thyroid dysfunction/cardiac disease was 2.3 times more than healthy mothers $(P<0.01)$.

Conclusion: With respect to the above and due to the role and importance of mother-infant health in community health, it is necessary that the health-care system improve health education with regard to the appropriate number of pregnancies, diagnose and cure disease during pregnancy, especially diabetes and cardiovascular disease (hypertension and/or eclampsia), and recognize pregnant mothers with mental pressure or lack of sufficient support and help them.

Keywords: premature labor, general health, psychosomatic disorder, number of pregnancies

\section{Introduction}

Preterm birth is defined by the World Health Organization (WHO) as birth of an infant prior to 37 weeks' (259 days') completed gestation. ${ }^{1}$ The most important outcome of preterm labor is a premature neonate. Previous studies have estimated that $11.1 \%$ of all live births are preterm worldwide, ranging from about 5\% in several European countries to $18 \%$ in some African countries. ${ }^{2}$

Prematurity is the most prevalent cause of mortality in infants. ${ }^{3}$ Based on global reports, $60 \%-80 \%$ of neonatal mortalities (not accompanied by congenital abnormalities) occur in premature infants, resulting in asphyxia in the first week and septicemia in the fourth week. ${ }^{4}$

Studies have revealed that mothers' education, age over 36, history of having a premature infant, multiparity, mother hypertension, infant diabetes, oligohydramnios 
polyhydramnios, placenta previa, anatomic abnormality of uterus, history of organic disorder (cardiac, renal, thyroid), and blood group type A have significant correlation with recurrence of premature labor. ${ }^{5,6}$

In addition to problems and expenses related to prematurity of infants, preterm labor may cause cerebral palsy, infection, fetal or neonatal death, and serious problems in personal skills development of children at school age. ${ }^{5}$

Every year, 3.6 million neonatal mortality cases are observed around the globe, of which 99 percent take place in developing countries. ${ }^{7}$ In Iran, the most important causes of newborn death were prematurity $(63.24 \%)$, constituting nearly two-thirds of neonatal mortalities. ${ }^{8}$

In a number of studies, the most prevalent risk factors leading to premature birth consist of insufficient pregnancy care (52\%), mothers' age being under 20 (34.7\%), third-trimester hemorrhage (23.4\%), and eclampsia and preeclampsia (13.1\%). The most prevalent complications of preterm labor reported in infants include septicemia (66.7\%), hyperbilirubinemia (58.8\%), asphyxia (26.8\%), and complications regarding the hyaline membrane $(23.3 \%){ }^{9,10}$

Based on statistics of the vice-chancellor for health of Yasuj University of Medical Sciences, neonatal mortality proportion is 15 in 1000 live births, prematurity being among the major causes of mortality. With respect to the importance of neonatal mortality and the fact that no effective treatment is currently available, the incidence of preterm labor could be prevented by effective diagnosis and preventative factors. This study was aimed to determine the most important factors related to preterm labor in Yasuj, Iran.

\section{Materials and methods}

This case-control study was conducted in the maternity ward of Imam Sajjad Hospital, the obstetrics and gynecology center of Yasuj, in 2010. In this retrospective research, the study population was drawn from mothers who had been referred to this center for delivery and were willing to participate in the study. Among eligible samples and with attention to last menstruation period, the mothers who had delivered an infant prior to 37 weeks' (259 days') completed gestation were selected as the case group, and for each sample in the case group, one mother with full-term labor who delivered an infant within or after 37 weeks (259 days) completed gestation were selected by using clipper-matched sampling to make up the control group. ${ }^{11}$

In this study, in addition to such ethical considerations as voluntary participation in research, security of data, and consent, etc, ethical committee approval was obtained from
Yasuj University of Medical Sciences, then data were collected using a researcher-made questionnaire consisting of demographic and associated factors regarding preterm labor and the Standard General Health Questionnaire.

The 28-item General Health Questionnaire assesses four subscales of somatic symptoms: anxiety, insomnia, social dysfunction, and severe depression of subjects. This questionnaire had four answer choices of "no," "a little," "a lot," and "very much." Marks allocated to the answers were 0, 1, 2, and 3, respectively (Likert method). The maximum score in this questionnaire is 84 . Based on the questionnaire, participants were divided into healthy or nonhealthy groups. ${ }^{12}$

In addition, other sources such as documents available in the pregnancy care records, urban and rural health centers, hospital medical records, and other available documents, such as insurance booklets, were used for increasing data accuracy.

In this study, mothers' age, education, job, weight, place of residence, income, nutrition status, body mass index, number of deliveries, number of pregnancies, history of abortion, stillbirth history, twin or multiplex pregnancy, history of smoking, alcohol usage, pregnancy excess weight, general health status, history of preterm labor, exposure to radiation, and history of diabetes mellitus/thyroid dysfunction/

Table I Comparison between variables in preterm and full term labor using univariate analysis

\begin{tabular}{lllll}
\hline Variable & $\chi^{2}$ & df & P-value & Sig \\
\hline Mothers' age & 1.29 & $\mathrm{I}$ & 0.21 & $\mathrm{NS}$ \\
Education & 3.82 & 2 & 0.14 & $\mathrm{NS}$ \\
Job & 6.24 & $\mathrm{I}$ & 0.1 & $\mathrm{NS}$ \\
Height & $\mathrm{I} .29$ & $\mathrm{I}$ & 0.21 & $\mathrm{NS}$ \\
Weight & 2.19 & $\mathrm{I}$ & 0.17 & $\mathrm{NS}$ \\
Place of residence & 65.3 & 2 & 0.16 & $\mathrm{NS}$ \\
Income & 6.79 & $\mathrm{I}$ & 0.09 & $\mathrm{NS}$ \\
Nutrition status & 2.19 & $\mathrm{I}$ & 0.17 & $\mathrm{NS}$ \\
BMI & $5.4 \mathrm{I}$ & 2 & 0.06 & $\mathrm{NS}$ \\
Number of deliveries & 5.98 & 2 & 0.06 & $\mathrm{NS}$ \\
Number of pregnancies & $9.9 \mathrm{I}$ & $\mathrm{I}$ & 0.001 & $\mathrm{~S}$ \\
History of abortion & 2.19 & $\mathrm{I}$ & 0.17 & $\mathrm{NS}$ \\
Stillbirth history & 2.29 & $\mathrm{I}$ & 0.13 & $\mathrm{NS}$ \\
Twin or multiplex pregnancy & 2.43 & $\mathrm{I}$ & 0.11 & $\mathrm{NS}$ \\
Smoking & $\mathrm{I} .09$ & $\mathrm{I}$ & 0.32 & $\mathrm{NS}$ \\
Alcohol usage & 0.79 & $\mathrm{I}$ & 0.49 & $\mathrm{NS}$ \\
Pregnancy excess weight & 6.79 & $\mathrm{I}$ & 0.09 & $\mathrm{NS}$ \\
General health status & $\mathrm{I} 8.6$ & 2 & 0.002 & $\mathrm{~S}$ \\
History of preterm labor & 2.29 & $\mathrm{I}$ & 0.13 & $\mathrm{NS}$ \\
Exposure to radiation & 2.19 & $\mathrm{I}$ & 0.17 & $\mathrm{NS}$ \\
History of diabetes mellitus/ & 9.64 & 3 & 0.004 & $\mathrm{~S}$ \\
thyroid dysfunction/cardiac & & & & \\
disease of mothers & & & & \\
\hline Abreviations & & & \\
\hline
\end{tabular}

Abbreviations: df, degrees of freedom; Sig, significance; NS, not significant; $\mathrm{S}$, significant; BMI, body mass index. 
Table 2 Results of multivariate analysis using logistic regression model

\begin{tabular}{llllll}
\hline Variable & Beta coeff & Wald statistic & P-value & OR & 95\% Cl for OR \\
\hline Number of pregnancies & -1.40 & 12.86 & 0.000 & 0.18 & $0.07-0.46$ \\
Mothers' general health & -1.06 & 7.36 & 0.007 & 0.35 & $0.16-0.75$ \\
History of diabetes mellitus/thyroid & -0.82 & 8.99 & 0.003 & 0.44 & $0.26-0.75$ \\
dysfunction/cardiac disease of mothers & & & & & \\
\hline
\end{tabular}

Note: The level of determining coefficient for the above model was estimated to be $60 \%$.

Abbreviations: coeff, coefficient; $\mathrm{OR}$, odds ratio; $\mathrm{Cl}$, confidence interval.

cardiac disease of mothers were compared between the two groups.

Finally, after discarding imperfect questionnaires, collected data of 52 subjects of the case group and the same number in the control group were analyzed using the SPSS (IBM, Armonk, NY) statistical software package.

Mean, standard deviation, frequency, percentage, univariate analysis, multivariate analysis using logistic regression model, odds ratio, and 95\% confidence interval were used for analytic inference. In this study, the significant level for interpretation of results was 0.05 .

\section{Results}

Among the 5400 live birth infants in Yasuj at 2010, 130 infants were premature $(2.4 \%)$. The mean age of the mothers was $26.1 \pm 4.3$ years, and their average height was $160.79 \pm 5.91 \mathrm{~cm}$. A total of $50.5 \%$ of mothers had an education level less than diploma, $32.3 \%$ had a diploma, and $17.2 \%$ had higher education than a diploma.

As Table 1 demonstrates, there were not statistically significant differences between the case and control groups in any of the investigated variables except for number of pregnancies, general health status, and history of diabetes mellitus/thyroid dysfunction/cardiac disease of mothers.

Table 2 consists of results of a multivariate analysis using logistic regression model and shows that there were significant correlations between number of pregnancies (odds ratio [OR], 0.18; 95\% confidence interval [CI], 0.07-0.46), general health status (OR, $0.35 ; 95 \% \mathrm{CI}, 0.16-0.75)$, and previous history of diabetes mellitus/thyroid dysfunction/ cardiac disease of mothers (OR, $0.44 ; 95 \% \mathrm{CI}, 0.26-0.75)$ of the case and control groups $(P<0.01)$. Because the reference group in these analyses was the case group, the above results show that the preterm labor risk in women with two or more pregnancies is 5.5 times $(1 / 0.18)$ higher than in women with less than two pregnancies, its risk in women with low general health status is 2.9 times $(1 / 0.35)$ higher than in women with normal general health status, and the preterm labor risk in women with history of diabetes mellitus/thyroid dysfunction/cardiac disease is 2.3 times $(1 / 0.44)$ higher than in healthy mothers.

\section{Discussion and conclusion}

Three variables, being number of pregnancies, mothers' general health, and diabetes mellitus/thyroid dysfunction/cardiac disease of mothers, accompanied risk of preterm labor. According to various studies, the role of numerous risk factors such as lifestyle, smoking, insufficient nutrition, and low weight gain during pregnancy have been recognized in preterm labor and intrauterine growth retardation. Other risk factors in previous studies include addiction to narcotics, use of alcohol, ambient poisons, prolonged standing, intensive work, activity, stress, lack of social support, low age of mother, illiteracy and poverty, first pregnancy, multiparity, hydraminus, abdominal surgery during pregnancy, abnormal uterus fever, disease during pregnancy, bleeding during pregnancy, history of pyelonephritis, history of having a low-weight infant, history of abortion, and hypertension. ${ }^{13-15}$

Most studies have considered the role of spiritual mental factors as important in growth and development of the fetus. Regarding the findings of the present study, a significant correlation was observed between incidence of preterm labor and the general health status of mothers (OR, 0.347), which is consistent with the findings of Ghosh et al, who reported that fear of delivery and chronic stress increase the risk of preterm labor (OR, 1.46).${ }^{16}$ In her study, Rondo pointed out the role of stress at the beginning of pregnancy, anxiety and depression in the last months of pregnancy, and their effects on premature labor. ${ }^{17}$

Various mechanisms have been suggested for this correlation. Direct correlation between premature delivery and mothers' mental health status (level of stress, anxiety, depression) may be correlated with the release of catecholamines. As the result of the release of catecholamines, and therefore reduction of placenta blood circulation, reduction of oxygen and nutrients in the fetus lead to disorders in fetal growth and premature labor. ${ }^{15,18}$ 
Given that stress increases corticotropin-releasing hormone, cortisol, and plasma levels, it may contribute to an increase in uterine contractions and consequently premature labor. ${ }^{19}$

Another finding of this study was a significant correlation between the number of pregnancies and incidence of premature labor. Although the etiology in many cases is unknown and idiopathic, the findings of the present study are contrary to the results of studies performed by Shah ${ }^{20}$ and Babinszki et al, ${ }^{21}$ as those studies did not recognize multiparity and grand parity as a cause of increased risk of premature labor, but are consistent with the results of Guo et al, ${ }^{22}$ who also mentioned this point and stated that number of pregnancies is a risk factor for premature labor and found that prevalence of premature labor in women is $6 \%$ regarding the first pregnancy, $4.3 \%$ with the second pregnancy, $4 \%$ with the third, and 5.7\% with fourth pregnancies. Also Reime et al concluded that the risk of premature labor is increased by second pregnancy in comparison to the first. ${ }^{23}$

Eclampsia and pregnancy hypertension is a status that is present in 5\%-7\% of all deliveries, and is correlated with main fetal disease and premature labor. ${ }^{24}$ Based on the findings of present study, a significant correlation was observed between the incidence of premature labor and history of diabetes mellitus/thyroid dysfunction/cardiac disease of mothers. This finding was consistent with the findings of a study conducted by Shingairai et al, in which a significant correlation was found between eclampsia during pregnancy and premature labor. ${ }^{25}$

Also in conformity with the findings of the present study, Covarrubias et al recognized the correlation between mothers' diseases such as eclampsia and preeclampsia and diabetes during pregnancy with premature labor. ${ }^{6}$

Although maternal smoking is a well-accepted risk factor and use of alcohol can cause preterm birth, in this study there was no evidence for these effects on preterm labor, because smoking and alcohol drinking are very rare in the study population due to culture and religion.

In this study, some important variables such as consanguineous marriage were missed in the questionnaire design. This was a limitation of this study, so we suggest other researchers attend to this in future studies regarding factors related to preterm labor.

Generally, with respect to the above and due to the role and importance of mother-infant health in community health, it is necessary that the health-care system improve health education with regard to appropriate number of pregnancies, diagnose and cure disease during pregnancy, especially diabetes and cardiovascular disease (hypertension and/or eclampsia), and recognize pregnant mothers with mental pressure or lack of sufficient support and help them.

\section{Acknowledgment}

This article is based on a student's thesis. We express our gratitude and appreciation to the Deputy of Research and Technology of Yasuj University of Medical Sciences for confirmation, approval, and providing expenses for the present research.

\section{Disclosure}

The authors report no conflicts of interest in this work.

\section{References}

1. Rafael TJ, Hoffman MK, Leiby BE, Berghella V. Gestational age of previous twin preterm birth as a predictor for subsequent singleton preterm birth. Am J Obstet Gynecol. 2012;206(2):156.e1-156.e6.

2. Blencowe H, Cousens S, Oestergaard M, et al. National, regional, and worldwide estimates of preterm birth rates in the year 2010 with time trends since 1990 for selected countries: a systematic analysis and implications. Lancet. 2012;379(9832):2162-2172.

3. Institute of Medicine (US) Committee on Understanding Premature Birth and Assuring Healthy Outcomes, Behrman RE, Butler AS. Preterm Birth: Causes, Consequences, and Prevention. Washington: National Academies Press; 2007.

4. Choi YY, Song ES, Kim YH, Song TB. Analysis of high-risk infant births and their mortality: ten years' data from Chonnam National University Hospital Medical School, Gwangju, Korea. Chonnam Med J. 2011;47(1):31-38.

5. O'Shea TM, Allred EN, Dammann O, et al. The ELGAN study of the brain and related disorders in extremely low gestational age newborns. Early Hum Dev. 2009;85(11):719-725.

6. Covarrubias LO, Aguirre GE, Chapuz JR, May AI, Velázquez JD, Eguiluz ME. Maternal factors associated to prematurity [Spanish]. Ginecol Obstet Mex. 2008;76(9):526-536.

7. Lawn JE, Kerber K, Enweronu-Laryea C, Cousens S. 3.6 million neonatal deaths - what is progressing and what is not? Semin Perinatol. 2010;34(6):371-386.

8. Hadavi M, Alidalaki S, Abedininejad M, Akhavan S. Etiologies and contributing factors of perinatal mortality: a report from southeast of Iran. Taiwan J Obstet Gynecol. 2011;50(2):145-148.

9. Gupta N. Perinatal mortality: an analysis of causes and strategies. Indian Med Assoc. 2011;109(4):245-246, 255-257.

10. Shrestha S, Dangol SS, Shrestha M, Shrestha RP. Outcome of preterm babies and associated risk factors in a hospital. J Nepal Med Assoc. 2010;50(180):286-290.

11. Bolajoko O, Olusany A. Full-term newborns with congenital microcephaly and macrocephaly in Southwest Nigeria. Int Health. 2012;4(2):128-134.

12. Nagyova I, Boudien K, Szilasiova A, Stewart RE, van Dijk JP, van den Heuvel WJA. General Health Questionnaire-28: psychometric evaluation of the Slovak version. Stud Psychol (Bratisl). 2000;42(4):351-361.

13. Stewart PJ, Nimrod C. The need for a community-wide approach to promote healthy babies and prevent low birth weight. Can Med Assoc J. 1993;149(3):281-285.

14. Engmann C, Walega P, Aborigo RA, et al. Stillbirths and early neonatal mortality in rural Northern Ghana. Trop Med Int Health. 2012;17(3):272-282.

15. Diallo FB, Diallo MS, Sylla M, et al. Premature delivery: epidemiology, etiologic factors, prevention strategies [French]. Dakar Med. 1998;43(1):70-73. 
16. Ghosh JKC, Wilhelm MH, Dunkel-Schetter C, Lombardi CA, Ritz BR. Paternal support and preterm birth, and the moderation of effects of chronic stress: a study in Los Angeles County mothers. Arch Womens Ment Health. 2010;13(4):327-338.

17. Rondo PHC. Maternal stress/distress and low birth weight, preterm birth and intrauterine growth restriction - a review. Curr Womens Health Rev. 2007;3(1):13-29.

18. Graignic-Philippe R, Tordjman S. Effects of stress during pregnancy on infant and child development [French]. Arch Pediatr. 2009;16(10):1355-1363.

19. Chou IC, Lien HC, Lin HC, et al. The relationship of salivary and cord blood cortisol in preterm infants. $J$ Pediatr Endocrinol Metab. 2011;24(1-2):85-88.

20. Shah PS. Knowledge Synthesis Group on Determinants of LBW/PT births. Parity and low birth weight and preterm birth: a systematic review and meta-analyses. Acta Obstet Gynecol Scand. 2010;89(7):862-875.
21. Babinszki A, Kerenyi T, Torok O, Grazi V, Lapinski RH, Berkowitz RL. Perinatal outcome in grand and great-grand multiparity: effects of parity on obstetric risk factors. Am J Obstet Gynecol. 1999;181(3):669-674.

22. Guo LJ, Ye RW, Wang GX, Wang J, Li ZW, Ren AG. Birth weight distribution among premature infants and related social factors [Chinese]. Zhonghua Liu Xing Bing Xue Za Zhi. 2009;30(12):1243-1247.

23. Reime B, Schuecking BA, Wenzlaff P. Perinatal outcomes of teenage pregnancies according to gravidity and obstetric history. Ann Epidemiol. 2004;14(8):619.

24. Lazdam M, De la Horra A, Pitcher A, et al. Elevated blood pressure in offspring born premature to hypertensive pregnancy: is endothelial dysfunction the underlying vascular mechanism? Hypertension. 2010;56(1):159-165.

25. Shingairai AF, Siobán DH, Godfrey BW. Risk factors for prematurity at Harare Maternity Hospital, Zimbabwe. Int J Epidemiol. 2004;33(6):1194-1201.
International Journal of General Medicine

\section{Publish your work in this journal}

The International Journal of General Medicine is an international peer-reviewed open-access journal that focuses on general and internal medicine, pathogenesis, epidemiology, diagnosis, monitoring and treatment protocols. The journal is characterized by the rapid reporting of reviews, original research and clinical studies across all disease areas.

\section{Dovepress}

A key focus is the elucidation of disease processes and management protocols resulting in improved outcomes for the patient.The manuscript management system is completely online and includes a very quick and fair peer-review system. Visit http://www.dovepress.com/ testimonials.php to read real quotes from published authors.

Submit your manuscript here: http://www.dovepress.com/international-journal-of-general-medicine-journal 